\title{
Compliance with the clinical practice guidelines for the management of hepatitis $B$ and $C$ virus-related chronic liver disease: a survey based on hospitalized cirrhotic patients
}

\author{
Emanuele La Spada, ${ }^{1}$ Giuseppe Augello, ${ }^{2}$ Giovanni Anastasi, ${ }^{3}$ Aldo Spadaro,,${ }^{4}$ Francesco Taormina, ${ }^{5}$ \\ Felice Fiorello, ${ }^{6}$ Michele Stornello, ${ }^{7}$ Antonio Carroccio,${ }^{8}$ Umberto Castiglione, ${ }^{9}$ Rocco Siciliano, ${ }^{10}$ \\ Giuseppe Montalto, ${ }^{1}$ the Internal Medicine Collaborating Group
}

${ }^{1}$ Divisione di Medicina Interna ed Epatologia, AOUP P. Giaccone, Palermo; ${ }^{2}$ Divisione di Medicina Interna, Ospedale di Canicattì (AG); ${ }^{3}$ Divisione di Medicina Interna, Ospedale di Marsala (TP); ${ }^{4}$ Divisione di Medicina Interna, AOUP G. Martino di Messina; ${ }^{5}$ Divisione di Medicina Interna, Ospedale S. Antonio Abate, Trapani; ${ }^{6}$ Divisione di Medicina Interna, Ospedale Buccheri Le Ferla, Palermo; ${ }^{7}$ Divisione di Medicina Interna, Ospedale Umberto I di Siracusa; ${ }^{8}$ Divisione di Medicina Interna, Ospedale di Sciacca (AG); ${ }^{9}$ Divisione di Medicina Interna, Ospedale S. Elia, Caltanissetta; ${ }^{10}$ Divisione di Medicina Interna, Ospedale di Acireale (CT), Italy

\begin{abstract}
In recent years, significant progress has been made in furthering our knowledge of chronic liver disease (CLD) and evaluating the therapeutic approaches. These have been updated in the form of recommendations by international scientific societies. Through a retrospective analysis, this study aimed to verify whether these recommendations have been applied in real practice. The study design included data gathered from all patients consecutively hospitalized for decompensated liver cirrhosis during one year. A pre-made master form was used to record data on the patients' past knowledge of the etiology and management of their liver disease. As expected, hepatitis C virus (HCV) was the most frequent cause of CLD, while 41 cases were cryptogenic. In 69 of 263 patients with HCV infection, viral genotyping had been performed, although only 39 of these cases had been treated. Only 3 of 44 patients suffering from hepatitis B virus (HBV)-related liver cirrhosis had been treated in the past, while 11 patients were still being treated. Among the remaining patients, 15 were not aware that they had CLD and 15 had never been considered for antiviral treatment. In 81 cases, the disease had progressed to hepatocellular carcinoma, but only 19 patients had discovered the tumor following regular ultrasound screening. Thirty-seven patients were receiving specific treatment consistent with the stage of their disease. The management of HBV- and HCV-related CLD in Sicily is far from optimal, and although the natural history and management practices of these diseases are well known, this knowledge is a long way from being applied in our daily practice.
\end{abstract}

Correspondence: Giuseppe Montalto, Medicina Interna, Università di Palermo, via del Vespro 141, 90127 Palermo, Italy. Tel. +39.91.6552991 - Fax: +39.91.6552948.

E-mail: giuseppe.montalto@unipa.it

Key words: chronic liver disease, clinical practice guidelines, antiviral therapy, ultrasound screening, compliance.

Conflict of interests: the authors declare no potential conflict of interests.

Acknowledgments: we would also like to acknowledge the collaboration of the other members of the Internal Medicine Collaborating Group, Walter Granà (Palermo), Claudia Fantucchio (Canicattì), Andrea Ditta, (Trapani), Roberto Risicato (Siracusa), Salvatore Leotta (Acireale).

This work is licensed under a Creative Commons Attribution NonCommercial 3.0 License (CC BY-NC 3.0).

CCopyright E. La Spada et al., 2013

Licensee PAGEPress, Italy

Italian Journal of Medicine 2013; 7:107-112

doi:10.4081/itjm.2013.107

\section{Introduction}

In recent years, significant progress has been made in furthering our knowledge of chronic liver disease (CLD) and determining therapeutic approaches. Among the developments in this field are the identification of the hepatitis $\mathrm{C}$ virus (HCV) nearly two decades $\mathrm{ago}^{1}$ and the availability of different treatment options. ${ }^{2}$ In addition, a group of CLD patients has gradually emerged, presenting with non-alcoholic fatty liver disease (NAFLD), ${ }^{3}$ which is predominantly linked to Western society and its affluent lifestyle. In Italy, the prevalence of liver disease related to hepatitis B virus (HBV) infection has significantly decreased. ${ }^{4}$ For some years now, patients have been able to benefit from medical interventions that may reduce the risk of developing end-stage liver disease and also improve long-term survival, even if this goal is often achieved with continuous antiviral treatment. ${ }^{5}$ In the meantime, the World Health Organization and other international scientific societies have issued and updated a number of recommendations to increase the awareness of 
$\mathrm{HBV}$ and $\mathrm{HCV}$ infections among health care providers and to contribute to the prevention and management of these infections..$^{6-8}$ Unfortunately, despite attempts to ensure that the medical community has access to this growing knowledge there is still a high frequency of patients with liver cirrhosis who require hospitalization on Internal Medicine wards because of complications, including the development of hepatocellular carcinoma (HCC), without any evidence of previous management of their liver disease.

It is now widely known from the natural history of CLD (including all of the forms previously mentioned, with viral etiology being the most common) that the development of liver cirrhosis is, in most cases, preceded by a long time lapse, during which cytolysis and remission phases alternate until a state of complete fibrosis is reached; this can take decades. ${ }^{9,10}$ During this long period of time, medical intervention can play a crucial role in curing, or at least limiting, the progression of liver damage if it is in the early stages. Furthermore, for the clinical management of patients who already have cirrhosis (Child-Pugh's classes A and B), the consolidated literature largely recommends that these patients undergo ultrasound screening every six months following an early diagnosis of HCC..$^{11}$ It is also true that, in some cases, CLD can be clinically indolent, with liver function tests remaining normal for a long period. But the frequency of patients who required hospitalization for decompensated CLD and had no knowledge of their disease or previous disease management obviously raises a series of questions. For this reason, we decided to perform a survey on a number of Internal Medicine wards in Sicily to verify whether the recommended guidelines on the different types of CLD are being applied in real practice.

\section{Materials and Methods}

This study included patients hospitalized on $10 \mathrm{Si}-$ cilian Internal Medicine wards, chosen on the basis of territorial distribution, including two centers from Palermo (the main city in the region) and one from each province of the island.

The study design involved gathering data from all of the patients consecutively hospitalized for decompensated liver cirrhosis during one year, from June 2010 to May 2011. In addition to these patients, we included patients hospitalized for other reasons (decompensated diabetes mellitus, heart disease, etc.) who also presented with liver cirrhosis. Patients were included only once, at their first clinical observation, even if they were hospitalized more than once during the study period. A premade master form was used to gather demographic data on all the patients. The form included the cause of hospitalization, etiology of CLD, knowledge of being a chronic $\mathrm{HBV}$ or $\mathrm{HCV}$ carrier, history of having been treated with antiviral drugs, the type of treatment, the response to treatment, inclusion in a semiannual ultrasound screening program for the early detection of HCC, and the type of treatment for HCC.

The diagnosis of liver cirrhosis was made on the basis of a previous liver biopsy showing a variable degree of liver damage and, upon inclusion in the study, on the basis of clinical, biochemical and instrumental data (ultrasound, computed tomography, transient elastography or magnetic resonance). The etiology of liver disease was based on the positive identification of viral markers (anti-HCV, HBsAg or $\mathrm{HBeAg}$ ). Alcohol was considered responsible for liver disease when consumption (either past or present) exceeded $40 \mathrm{~g} /$ day for men or $30 \mathrm{~g} /$ day for women for at least five years. NAFLD was diagnosed for liver disease with negative viral markers, absent or minimal alcohol consumption, and the presence of metabolic syndrome stigmata (e.g. diabetes mellitus, arterial hypertension, dyslipidemia, and obesity), either currently or in the past. Autoimmune liver disease included liver cirrhosis with a past history of autoimmune hepatitis, primary biliary cirrhosis or primary sclerosing cholangitis, while cryptogenic liver disease included those forms in which the common causes of CLD could be excluded. To diagnose HCC, a biopsy of the lesion or imaging techniques were used, in accordance with well-accepted criteria. ${ }^{11}$ Whatever the causes, all patients were requested to indicate how long they had been aware of their disease, what treatment(s) had been administered in the past or were currently being followed, and adherence and response to the given treatment.

Because this study included only data gathered from an anonymous form from the hospital cards, approval from the Ethical Committee was not required. All of the patients did, however, give their informed consent to participate in the study.

\section{Statistical analysis}

Continuous variables are summarized as the mean $\pm \mathrm{SD}$, and categorical variables are given as the absolute and relative frequencies. No comparisons of these data were performed because this was a study of descriptive epidemiology.

\section{Results}

In total, 429 patients were recruited during the oneyear period. They included 248 males and 181 females with a mean age of $68.8 \pm 11.5$ years. Their distribution according to the Child-Pugh class is shown in Table 1.

The main causes of hospitalization are shown in Figure 1. The most frequent cause was ascites (at the first occurrence in 81 cases). It alone was responsible for almost one-third of all the hospitalizations; when 
associated with the other causes, ascites was determined to be responsible for three-quarters of all hospitalizations. Porto-systemic encephalopathy was the second cause $(25 \%)$, followed by gastro-intestinal hemorrhage (18\%). Eighteen percent of hospitalizations were for causes other than decompensated liver disease, but all of these patients also presented with associated liver cirrhosis.

Figure 2 shows the etiologies of CLD included in the study. As expected, HCV, even when considered alone, was the most frequent agent (50.4\%), followed by alcohol consumption (11.7\%). HBV alone was responsible for $4.2 \%$ of all cases. Finally, 41 cases $(9.6 \%)$ were found not to have a risk factor for liver disease and were labeled as cryptogenic, but in 35 cases, the viral markers had never been assayed. Other $(11.9 \%)$ indicated some forms of autoimmune pathologies and all end-stage liver disease from nonalcoholic steatohepatitis, with many of the cases showing stigmata of metabolic syndrome.

In 26 of 263 patients with HCV infection, antiHCV positivity was established during current hospitalizations, and only 69 cases had viral genotyping performed in the past (64 G1b; 3 G1c; 1 G2c and 1 G3). Only 39 of these patients had been previously treated with antiviral therapies [standard/pegylated in- terferon (IFN) \pm ribavirin], 36 patients were nonresponders (NR), and 3 patients had previously relapsed and were not being re-treated. Therefore, most patients (198 of 237 were aware of their positivity) had never received antiviral therapy.

Only 3 of 44 patients with HBV-related liver cirrhosis had been treated with standard IFN in the past but were NR, and 11 patients (one of whom had previously been treated with IFN) were being treated at time of the study with a nucleotide/nucleoside analogue. Among the remaining 31 patients, 18 had not been aware that they were suffering from CLD until the present hospitalization, and the other 13 , despite being aware themselves, had never been considered for antiviral treatment. It was not possible to obtain data on anti- $\delta$ positivity for any of the cases.

Even alcohol abuse, which is usually considered to be less frequent in southern Italy compared to northern Italy, was a prevalent etiology of liver cirrhosis, only being responsible for 50 cases and involved in 83 cases in association with viral etiologies. Moreover, the number of cases with alcohol-induced cirrhosis may be underestimated because of the widespread social tendency in southern Italy to deny alcohol consumption.

In $81(18.8 \%)$ of our hospitalized cirrhotic patients

Table 1. Age (mean \pm SD), gender and Child-Pugh's class of the patients included in the study.

\begin{tabular}{lccc}
\hline & No. & Age (years) & Child-Pugh A/B/C \\
\hline Total & 429 & $68.8 \pm 11.5$ & $72 / 178 / 179$ \\
\hline Males & 248 & $66.5 \pm 13.1$ & $34 / 110 / 104$ \\
\hline Females & 181 & $71.3 \pm 9.8$ & $38 / 68 / 75$ \\
\hline
\end{tabular}

A, B, C, Child-Pugh's classes.

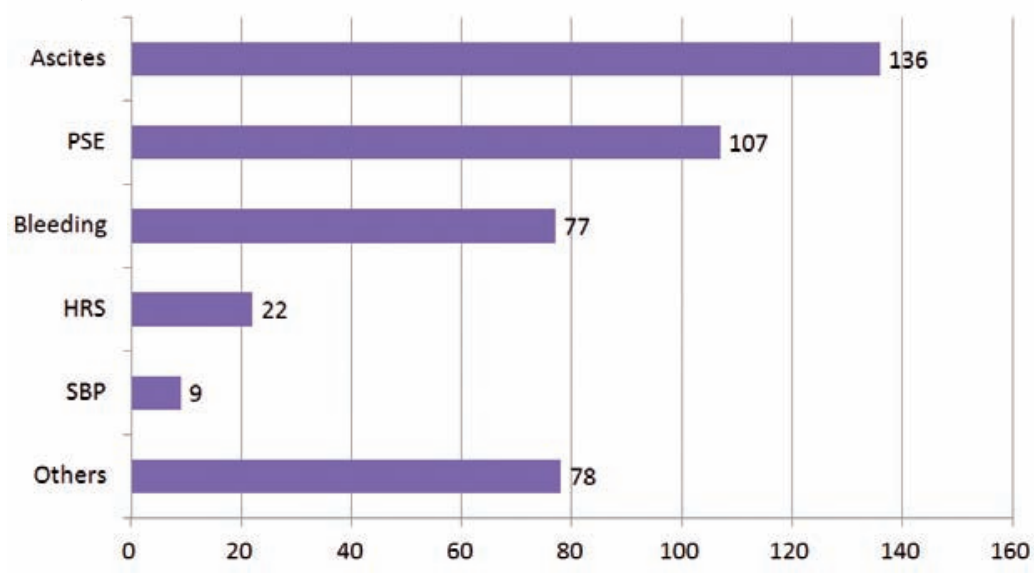

Figure 1. Main causes of hospitalization in liver cirrhosis patients included in the study. PSE, portal systemic encephalopathy; HRS, hepato-renal syndrome; SBP, spontaneous bacterial peritonitis. 
(56 males, 25 females; mean age $72.2 \pm 8.0$ years), the disease had progressed to HCC. Only in 19 of these patients $(23 \%)$ had the tumor been discovered following regular ultrasound screening; in the other cases, the diagnosis was based on signs of progression of liver cirrhosis. Based on the three Child-Pugh's classes, A, B and $C$, they were distributed as 13,33 and 35 patients, respectively. The etiology of these cases (Figure 3) was similar to that of liver cirrhosis in general, with a clear prevalence of HCV. Only 37 cases (45.7\%) had received specific treatment: one patient had undergone resection, 7 patients had radiofrequency thermal ablation (RFTA), 20 patients had transarterial chemoembolization (TACE), 5 patients had RFTA+TACE and 4 patients were being treated with sorafenib.

\section{Discussion and Conclusions}

This retrospective study aimed to evaluate patient awareness of the etiology and previous management of CLD in a group of patients hospitalized for end-stage liver disease to assess compliance with scientific recommendations. For the evaluation, we took into consideration the following data that has been confirmed in the literature: i) the progression from viral infection to liver cirrhosis takes roughly two decades; $;, 10$ and ii) the most frequent etiological agent, $\mathrm{HCV}$, has been known for at least 20 years, ${ }^{1}$ and although a specific treatment has been available since that time, an acceptable response has only been achieved more recently. This study, however, also allowed us to determine the main causes of hospitalization of patients with liver cirrhosis and its etiology in our region. As this is a retro-

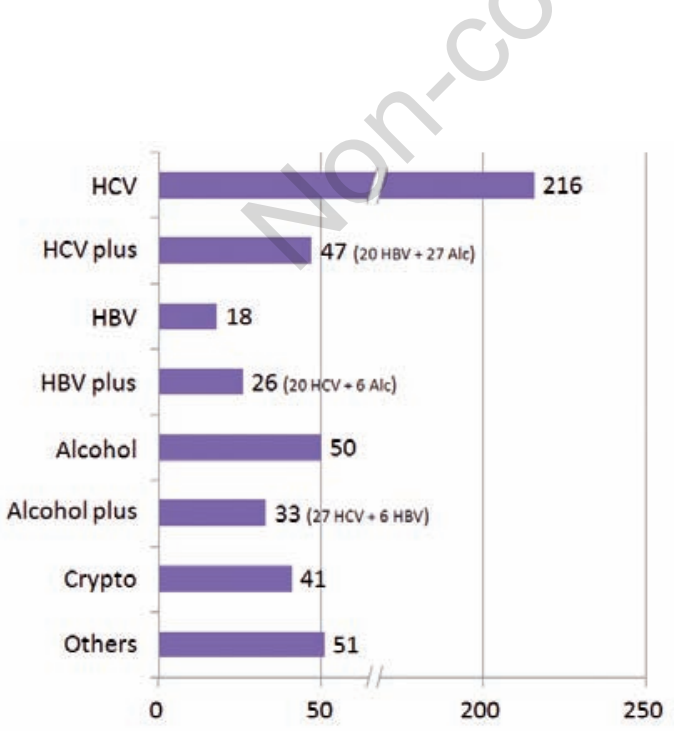

Figure 2. Main etiology of liver cirrhosis in patients included in this study. Hepatitis $\mathrm{C}$ virus (HCV) plus, HCV plus hepatitis B virus (HBV) or alcohol; HBV plus, HBV plus HCV or alcohol; alcohol plus, alcohol plus HBV or HCV. spective study, there is a limitation to its accuracy. What emerged was a picture of the causes of hospitalization for decompensated liver cirrhosis similar to that reported in the literature, with ascites being the principal cause. ${ }^{12}$ However, the cases of spontaneous bacterial peritonitis may be underestimated because of the trend in Sicily to only rarely perform diagnostic paracentesis for its detection.

HCV was confirmed to be the most frequent etiology, although the result was slightly lower than what we had reported previously, considering that viral markers had never been assayed for some patients. The same decrease was observed for HBV, while the frequency of alcohol abuse had clearly doubled. Another positive finding is that the mean age of the patients with cirrhosis in the present study was higher than the results of a previous study $(59.4 \pm 10.9) .{ }^{13}$

In the final analysis, there were not very many cases of patients with cirrhosis with an uninvestigated etiology, but an effort should be made to reduce this number to zero. What is quite surprising is that despite the high awareness of anti-HCV positivity, a high number of patients had not undergone antiviral treatment. There are various possible reasons for this lack of treatment: i) different recommendations in the past for patients with persistently normal transaminases or compensated liver cirrhosis; ii) patient refusal, which is quite frequent in $\mathrm{HCV}$-infected subjects in southern Italy: ${ }^{14}$ or iii) low eligibility rate for treatment due to comorbidities. ${ }^{15}$ Finally, in other reports, a lack of diagnosis was the first reason why patients were not treated, ${ }^{16}$ for which no justification is given.

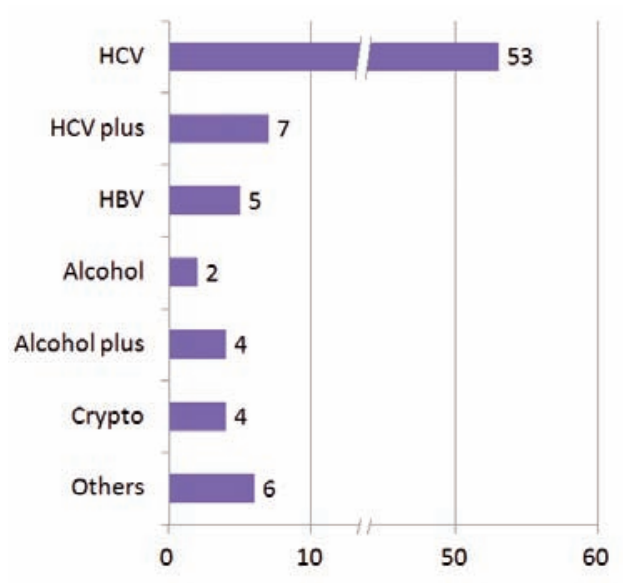

Figure 3. Main etiology of liver cirrhosis in patients who progressed to hepatocellular carcinoma. Hepatitis $\mathrm{C}$ virus (HCV) plus, HCV plus hepatitis B virus (HBV) or alcohol; HBV plus, HBV plus HCV or alcohol; alcohol plus, alcohol plus HBV or HCV. 
An interesting finding in our HCV positive patients with cirrhosis was that all of the patients $(n=39)$ treated in the past with the current therapies (i.e. standard or pegylated IFN+ribavirin) were either nonresponders $(n=36)$ or relapsed patients $(n=3)$ who were no longer being treated. This finding confirms that effective antiviral treatment eradicates the virus, leading to a definitive recovery from CLD, and that liver disease progresses in very few patients after sustained viral response. ${ }^{17}$ The same recommendation can be made even more strongly in HBV-related cases of liver cirrhosis. In fact, not only did a large number of patients not receive adequate antiviral treatment in the past, but also these patients were not receiving the current treatment recommended by our national guidelines at the time of hospitalization. ${ }^{18}$ However, it is also important to underline that many patients were unaware of their infection until the symptoms of liver cirrhosis had manifested. Unfortunately, these are not isolated findings. In fact, they are in line with other reports for the same geographical area, confirming that a strong communication campaign needs to be launched with the involvement of physicians to raise public awareness and promote a change in behavior. ${ }^{19}$ Even the results in our study could be a consequence of the downgrading of the HBV infection alert, ${ }^{20}$ the indolent clinical course of the disease,${ }^{21}$ or the fact that transaminase levels can remain within normal limits for a long period of time. ${ }^{22}$

Another important observation comes from the analysis of our data on patients who had progressed to HCC. While an increasing number of reports in the literature recommend that liver cirrhosis patients undergo regular ultrasound screening ${ }^{11,23,24}$ to diagnose $\mathrm{HCC}$ at an early stage (when they may benefit from curative treatment), after examining the treatment administered to our HCC patients, we observed very few early diagnoses. As a consequence, ultrasound screening has not been regularly performed. The distribution into Child-Pugh's categories and the type of therapies given to our patients are typical of an intermediate/advanced stage, when treatment is more palliative than curative. In fact, few patients had undergone resection or RFTA (both classical therapies suggested for small HCC) while most patients had TACE or medical treatment, in agreement with indications for intermediate or advanced HCC. ${ }^{25}$

The suggestions presented in this study and its limitations are neither novel nor isolated. Many reports in the literature evaluating compliance and the appropriateness of patient management with the scientific recommendations have shown similar results. There are only two examples in the field of hepatology: the recent survey in Italy on the treatment of chronic HBV-related hepatitis, in which a significant proportion of patients who were candidates for antiviral treat- ment were not being treated, showing an inadequate adherence to the current treatment recommendations, ${ }^{26}$ and the US study on the appropriateness of $\beta$-blockers in the prevention of gastro-esophageal variceal hemorrhage in cirrhosis, which showed a guideline adherence of only $54 \%{ }^{27}$

In conclusion, our data show that the main cause of hospitalization for decompensated liver cirrhosis on Internal Medicine wards is ascites and that HCV is still, by far, the most common etiological agent. However, despite being aware of their chronic infection, many patients did not follow the currently recommended antiviral therapy, even though this treatment could certainly have reduced or slowed the progression towards end-stage liver disease. In addition, in the case of HBV-related cirrhosis, the use of the new antiviral treatments, now recommended until the final stages of liver cirrhosis, is not adequately applied. Moreover, most of the current end-stage liver disease patients had not been followed up in a regular US screening surveillance program for HCC. Consequently, although the natural history and management of these diseases is already well known, this knowledge is far from being applied to daily practice.

\section{References}

1. Kuo G, Choo QL, Alter HJ, et al. An assay for circulating antibodies to a major etiologic virus of human nonA, non-B hepatitis. Science 1989;244:362-4.

2. Rosen HR. Clinical practice. Chronic hepatitis C infection. N Engl J Med 2011;364:2429-38.

3. Bellentani S, Tiribelli C. The spectrum of liver disease in the general population: lesson from the Dionysos study. J Hepatol 2001;35:531-7.

4. Sagnelli E, Stroffolini T, Mele A, et al. The importance of HCV burden of chronic liver disease in Italy: a multi center prevalence study of 9,997 cases. J Med Virol 2005;75:522-27.

5. Cowan ML, Thomas HC, Foster GR. Therapy for chronic viral hepatitis: current indications, optimal therapies and delivery of care. Clin Med 2011;11:184-9.

6. Grosheide P, Van Damme P. Prevention and control of hepatitis B in the community. In: Hallauer J, Kane M, McCloy E, Meheus A, Roure C, eds. Communicable diseases, Series no 1. Edegem: WHO Regional Office for Europe; 1996. pp 5-51.

7. Lok AS, McMahon BJ. Practice Guidelines Committee, American Association for the Study of Liver Diseases. Chronic hepatitis B. Hepatology 2001;34:1225-41.

8. Booth JC, O'Grady J, Neuberger J. The Royal College of Physicians of London and the British Society of Gastroenterology. Clinical guidelines on the management of hepatitis C. Gut 2001;49:11-21.

9. Tong MJ, El-Farra NS, Reikes AR, Co RL. Clinical outcomes after transfusion-associated hepatitis C. N Engl J Med 1995;332:1463-6.

10. Fattovich G. Natural history of hepatitis B. Sem Liv Dis 2003;23:47-58. 
11. Bruix J, Sherman M, Llovet JM, et al. Clinical management of hepatocellular carcinoma. Conclusions of the Barcelona 2000 EASL conference. J Hepatol 2001; 35:421-30.

12. Gines P, Quintero E, Arroyo V, et al. Compensated cirrhosis: natural history and prognostic factors. Hepatology 1987; 7:122-8.

13. Montalto G, Soresi M, Tripi S, et al. Frequenza di positività per anti-HCV in una popolazione di epatopatici siciliani. Ann It Med Int 1995;10:89-92.

14. Stroffolini T, Spadaro A, Guadagnino V, et al. Current practice of hepatitis $\mathrm{C}$ treatment in Southern Italy. Dig Liv Dis 2010;42:822-25.

15. Mariano A, Caserta C, Pendino GM, et al. Antiviral treatment for hepatitis $\mathrm{C}$ virus infection: effectiveness at general population level in a highly endemic area. Dig Liv Dis 2009;41:509-15.

16. Volk M, Tocco R, Saini S, Lock AS. Public health impact of antiviral therapy for hepatitis $\mathrm{C}$ in the United States. Hepatology 2009;50:1750-5.

17. Ng V, Saab S. Effects of sustained viral response on outcomes of patients with chronic hepatitis. Clin Gastroenterol Hepatol 2011;9:923-30.

18. Carosi G, Rizzetto M. Treatment of chronic hepatitis B. Recommendations from an Italian workshop. Dig Liv Dis 2008;40:603-17.

19. Ippolito AM, Niro GA, Fontana R, et al. Unawareness of HBV infection among inpatients in a Southern Italian hospital. J Vir Hepat 2011;18:206-11.

20. Raimondo $G$, Isgrò $G$, Caccamo $G$, et al. Is there a downgrading in the alert about the hepatitis $\mathrm{B}$ virus infection in Italy? Dig Liv Dis 2007;39:257-61.
21. Brunetto MR, Oliveri F, Coco B, et al. Outcome of antiHBe positive chronic hepatitis B in $\alpha$-IFN treated and untreated patients: a long term study. J Hepatol 2002; 36:263-70.

22. Papatheodoridis GV, Chrysantos N, Hadyziannis E, et al. Longitudinal changes in serum HBV-DNA levels and predictors of progression during the natural course of HBeAg-negative infection. J Vir Hepat 2008; 15:434-41.

23. Trevisani F, De NS, Rapaccini G, et al. Semi-annual and annual surveillance of cirrhotic patients for hepatocellular carcinoma: effects on cancer stage and patient survival (Italian experience). Am J Gastroenterol 2002;97: 734-44.

24. Sangiovanni A, Del Ninno E, Fasani P, et al. Increased survival of cirrhotic patients with a hepatocellular carcinoma detected during surveillance. Gastroenterology 2004;126:1005-14.

25. Cabrera R, Nelson DR. Review article: the management of hepatocellular carcinoma. Aliment Pharmacol Ther 2010;31:461-76.

26. Giannini EG, Torre F, Basso M, et al. A significant proportion of patients with chronic hepatitis B who are candidates for antiviral treatment are untreated. A region-wide survey in Italy. J Clin Gastroenterol 2009; 43:1001-7.

27. Carey E, Wakim-Fleming J, Lopez R, et al. National and regional conformity to the 2007 ACG/AASLD practice guidelines for prevention and management of gastroesophageal varices and variceal hemorrhage in cirrhosis. Am J Gastroenterol 2008;103:S1-35. 